\title{
Chapter 4 \\ Compact Ultrafast Oscillators and High Performance Ultrafast Amplifiers Based on Ytterbium-Doped Fibers
}

\author{
J Limpert, T. Eidam, M. Baumgartl, F. Röser, M. Plötner, \\ B. Ortaç, S. Nolte and A. Tünnermann
}

\begin{abstract}
This chapter reviews the fundamentals and achievements of ultrashort pulse generation and amplification in ytterbium-doped fibers. Compact and ultrastable passively mode-locked fiber oscillators represent an ideal seed source for high performance femtosecond fiber amplification systems, which have been scaled towards $\mathrm{kW}$-level average power and pulse energies well above the mJ-level. These laser systems will have significant impact in numerous scientific and industrial applications.
\end{abstract}

\subsection{Introduction and Motivation}

A number of important practical as well as fundamental research applications of ultrafast lasers appeared over the last decades, a trend initiated by the step from old dye-laser technology towards solid-state lasers. These high-power ultrafast solidstate lasers use small rods as the amplifier media, for instance, Titanium-doped sapphire as the most widespread one and have the potential to generate significantly higher pulse energies, higher powers and shorter pulse durations in combination

\footnotetext{
J. Limpert $(\bowtie) \cdot$ T. Eidam $\cdot$ M. Baumgartl $\cdot$ S. Nolte $\cdot$ A. Tünnermann

Friedrich-Schiller-Universität Jena, Institut Für Angewandte Physik, Max-Wien-Platz 1, 07743 Jena, Germany

e-mail: jens.limpert@uni-jena.de

F. Röser

Helmholtz-Zentrum Dresden-Rossendorf, Bautzner Landstraße 400, 01328 Dresden, Germany

M. Plötner

Fraunhofer IOF, Albert-Einstein-Straße 7, 07745 Jena, Germany

B. Ortaç

Bilkent Univ, UNAM Inst Mat Sci \& Nanotechnol, TR-06800, Ankara, Turkey

(C) Springer International Publishing Switzerland 2016

S. Nolte et al. (eds.), Ultrashort Pulse Laser Technology,

Springer Series in Optical Sciences 195, DOI 10.1007/978-3-319-17659-8_4
} 
with increased reliability than dye-lasers. However, these systems are difficult to scale in average power and suffer from low efficiencies, because direct diode pumping is not possible. Furthermore, the complexity of short pulse high energy Ti: sapphire lasers still constrain the employment of ultrafast laser technology in industrial environments. Material processing as one of the main driver applications relies on reliable, compact and cost effective pulsed sources.

In order to overcome thermo-optical effects, which limit the power scaling capability of these systems, several novel gain media designs, such as thin disk or slab, have been introduced $[1,2]$. However, due to the low single pass gain of these amplifier materials, very complex systems, such as regenerative amplification schemes, are required to obtain a reasonable output. Therefore the robustness, compactness and long-term stability are restricted in short pulse bulk solid-state laser systems.

Alternatively, forming the gain medium long and thin not only leads to outstanding thermo-optical properties, but also to a very high single pass gain. Fiberbased laser systems have the reputation to be immune against any thermo-optical problems due to their special geometry. The excellent heat dissipation is due to the large ratio of surface-to-active volume of such a fiber. The beam quality of the guided mode is determined by the fiber core design and is therefore powerindependent.

Due to the confinement of both the laser and pump radiation the intensity is maintained over the entire fiber length and is not limited to the Rayleigh length as it is the case in longitudinally pumped bulk lasers. The gain of the laser medium is determined by the product of pump light intensity and interaction length with the laser radiation in the gain medium. Therefore, the decisive product can be orders of magnitude higher in fibers than in other bulk solid-state lasers. This results in a very efficient operation of fiber laser systems exhibiting very high gain and low pump threshold values. Additionally, complete integration of the laser process in a waveguide provides an inherent compactness and long-term stability of fiber lasers.

In particular Ytterbium-doped glass fibers, which have a quantum defect of less than $10 \%$, can provide optical-to-optical efficiencies well above $80 \%$ and, therefore, low thermal load. These fiber laser systems are especially interesting for high power ultrashort pulse generation and amplification because of several unique properties [3]: Firstly, a broad emission spectrum allows for short pulse amplification. In ytterbium-doped glass fibers the amplification bandwidth of approximately $40 \mathrm{~nm}$ supports in principle pulses of durations as short as $\sim 30 \mathrm{fs}$. Furthermore, the absorption spectrum covers a wavelength range in which powerful diode lasers are commercially available. An additional point to note is that the long fluorescence lifetime ( $\sim 1 \mathrm{~ms}$ ) results in a high-energy storage capability. Excitedstate absorption of pump or signal radiation, or concentration quenching by ion-ion energy transfer processes does not occur with ytterbium, because only two energylevel manifolds are relevant for all optical wavelengths.

High power fiber lasers usually use the double-clad fiber concept (Fig. 4.1), invented in 1988 by Snitzer [4]. Such a double-clad fiber is characterized by a second waveguide, which is highly multimode, surrounding the active core. Into 


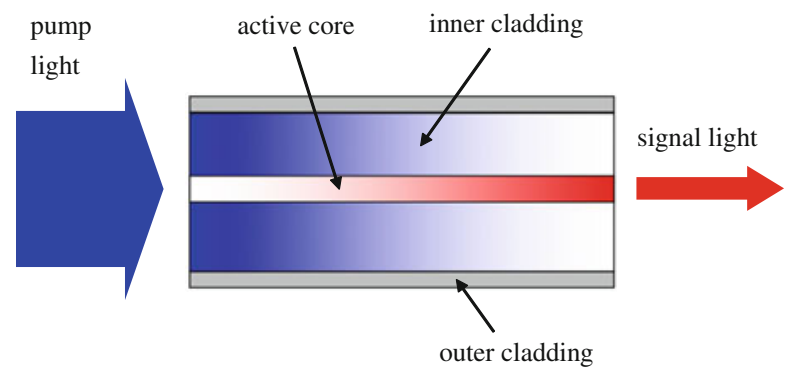

refractive index profile

Fig. 4.1 The double-clad fiber concept

this second waveguide, also called inner cladding or pump core, low brightness high power diode laser radiation can be launched. This pump light is gradually absorbed over the entire fiber length and is converted into high brightness high power laser radiation. Thus, double-clad rare-earth doped fibers can provide a highly efficient brightness improvement by pump-to-laser radiation conversion by the laser process itself.

All the mentioned properties make rare-earth-doped fibers superior to other solid-state laser concepts in a variety of performance categories. This has become obvious following several recent demonstrations of continuous-wave fiber laser systems exhibiting more than $10 \mathrm{~kW}$ of average power while maintaining an excellent beam quality [5].

\subsection{Fundamentals of Short Pulse Propagation in Passive and Active Fibers}

The fiber geometry itself is responsible for most of the outstanding properties of rare-earth-doped fibers that make them attractive gain media. However, this geometry also promotes nonlinear effects by making the light propagate under tight confinement over considerably long lengths. In fact, in the context of ultrashort pulse amplification, nonlinearity is mostly harmful and imposes performance limitations in fiber laser systems.

Nonlinear effects in fibers can be manifold [6]. The lowest-order nonlinear effects in standard optical fibers originate from the third-order susceptibility $\chi^{(3)}$. These effects can be divided into those related to an intensity-dependent refractive index and those resulting from stimulated inelastic scattering. Self-phase modulation (SPM), four-wave-mixing (FWM), and self-focusing all fall into the first category, whereas stimulated Raman scattering (SRS) and stimulated Brillouin scattering (SBS) are effects of the second category.

In general, the nonlinearity coefficients in silica glass fibers are intrinsically small. Both the nonlinear index coefficient $n_{2}$ and the gain coefficients of SRS and SBS are at least two orders of magnitude smaller than in other common nonlinear 
media [7]. Nevertheless, due to the large product of intensity and interaction length inside the fiber core, nonlinear effects can be observed at very low peak power levels and can basically limit the performance of pulsed rare-earth-doped fiber systems.

All the aforementioned nonlinear effects scale both with the light intensity in the fiber core and with the interaction length between the optical radiation and the nonlinear medium - that is, the fiber. Hence, to reduce the impact of nonlinearity, temporal and spatial scaling is required. Temporal scaling can be achieved by the well-known technique of chirped pulse amplification (CPA), which is schematically depicted in Fig. 4.2.

In this technique, ultrashort optical pulses from a mode-locked oscillator are stretched in time by a certain factor, which can be as large as 10,000 , by passing them through a dispersive delay line. Therefore, during amplification, the peak power of the pulses is considerably reduced, as are the nonlinear effects. After amplification, the stretched amplified pulses travel through a second dispersive delay line with the opposite sign of the stretcher dispersion, resulting in a recompression back to ultrashort pulse duration.

On the other hand, spatial scaling requires advanced fiber designs that present a large mode area of the actively doped core and an absorption length that is as short as possible, thus reducing the nonlinear interaction length. Consequently, there has been a pursuit of novel fiber designs with increased core dimensions that are still able to emit a stable fundamental mode. Conventional active step-index fibers with reduced numerical aperture, assisted by coiled or tapered sections to achieve singlemode operation, allow for core diameters of up to $40 \mu \mathrm{m}$. Rare-earth-doped photonic crystal fibers (PCFs) allow for significantly larger core sizes of up to $100 \mu \mathrm{m}$ [8-10] due to the significantly better control of the index step between a nanostructured core and the holey photonic crystal cladding. Due to the PCF cladding's design freedom, an additional functionality, such as polarizing or polarization maintaining properties, can be added [11]. An example of a large core ytterbiumdoped fiber is known as rod-type PCF. A cross section of the ultra-large mode area fiber is shown in Fig. 4.3. The $200 \mu \mathrm{m}$ inner cladding (pump waveguide) is

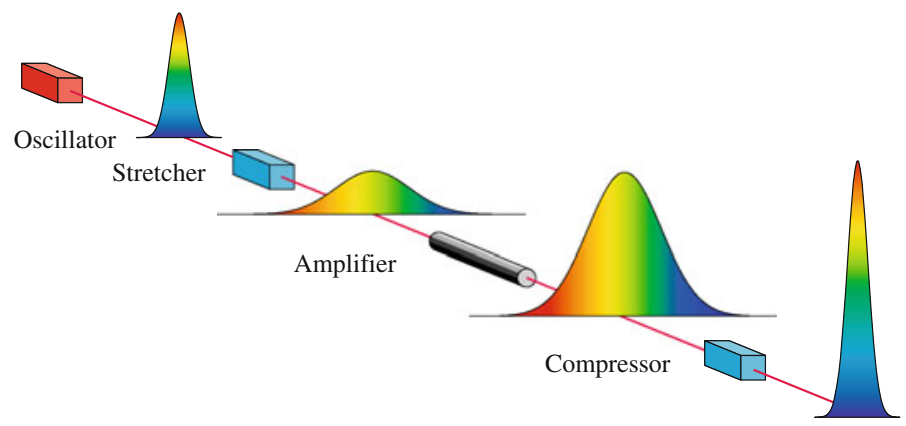

Fig. 4.2 Principle of chirped pulse amplification (CPA) 


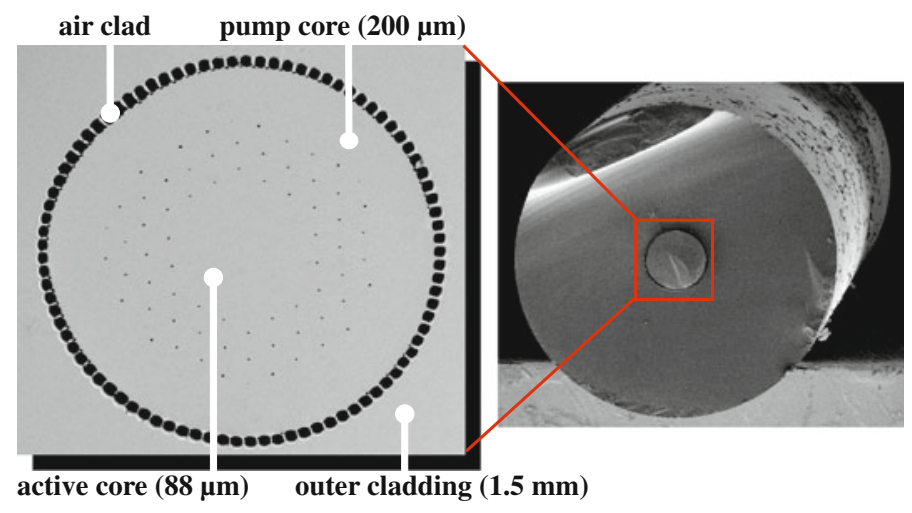

Fig. 4.3 Microscope image of a rod type photonic crystal fiber

surrounded by an air clad that consists of ninety $400 \mathrm{~nm}$ thick and $\sim 10 \mu \mathrm{m}$ long silica bridges. This structure provides a numerical aperture of $\sim 0.6$ at $976 \mathrm{~nm}$, allowing for an efficient coupling of multimode pump radiation into the fiber. Nineteen missing holes in the center of the fiber form the Yb/Al codoped active core region, which has a corner-to-corner distance of $88 \mu \mathrm{m}$. Three rings of small, carefully dimensioned airholes (pitch $\Lambda=\sim 14.9 \mu \mathrm{m}$; relative hole size $d /$ $\Lambda=\sim 0.1$ ) around this core provide the confinement of the radiation in the doped core. The mode area of the fundamental mode is as large as $4000 \mu \mathrm{m}^{2}$. The small ratio of pump core area to active core area results in an enhanced pump light absorption of about $30 \mathrm{~dB} / \mathrm{m}$ at $976 \mathrm{~nm}$. The entire inner structure is surrounded by a stiff 1.5-mm diameter fused silica outer cladding. This large outer cladding is primarily introduced to keep the fiber straight, thus preventing bend-induced losses or distortions of the weakly guided fundamental mode. Thus, in such a straight structure, the large mode area is accessible over the entire fiber length, which is not the case in conventional LMA fibers. In addition, the outer cladding makes the fiber mechanically robust on its own, so that no extra coating material is required; this allows for straightforward high-power extraction. By applying this kind of fiber, novel performance levels have been achieved in various operation regimes, ranging from femtosecond to nanosecond pulses [12-14].

\subsection{Compact All-Fiber Femtosecond Mode-Locked Lasers}

Sources of ultra-short laser pulses have become a versatile tool for an increasing number of industrial and scientific applications. Despite of the outstanding performance of passively mode-locked solid-state lasers, such systems have difficulties to step out the laboratory environment. This is mainly due to the bulky elements and free-space propagation inside the cavity. In contrast, fiber based sources give the possibility to generate laser radiation in a completely integrated manner, hence, 
they allow for the development of compact, inexpensive, misalignment-free and environmentally stable short-pulse lasers. Indeed, passively mode-locked singlemode rare-earth-doped fiber lasers are nowadays routinely operated and are entering the market to address real world applications.

Different operation regimes of mode-locked fiber lasers have been reported over the recent decades. Following conventional mode-locked oscillators, the most intuitive way is to run a fiber oscillator in the soliton regime, whereby modelocking is obtained using nonlinear polarization rotation inside the fiber or a semiconductor saturable absorber mirror to favour the pulsed mode. Soliton fiber lasers are built entirely from anomalous group-velocity dispersion (GVD) fiber and the pulse maintains its shape through the combined action of negative GVD and Kerr nonlinearity. However, the energy achievable in such a configuration is limited by the soliton area theorem to some tens of picojoules [15, 16]. Disobeying this theorem is leading to an excess of nonlinearity and finally is causing a pulse breakup. An increase of pulse energy from fiber oscillators can be achieved by stretching the pulse during its propagation. Thereby, the reduction of peak power keeps the nonlinear phenomena under control. This approach is referred to as stretched-pulse operation, in which the fiber laser comprises segments of large normal and anomalous GVD in the cavity [17]. The pulse width experiences large variations per cavity round trip, with a change in the chirp sign from positive at the end of the normal GVD segment to negative at the end of the anomalous GVD segment. In particular, with small net positive cavity dispersion, the pulse energy is larger than that produced in the soliton regime. Stretched pulse lasers with output energies from tens of picojoules to some nanojoules have been reported [18-22] before nonlinearity provokes instabilities. However, under certain circumstances nonlinearity can be advantageous. It has been shown that in fibers possessing normal GVD a pulse can propagate in a wave-breaking free manner despite strong nonlinearity [23]. In a fiber oscillator operated in the self-similar regime, the pulse accumulates a linear, or at least a monotonic, chirp, which is partially compensated at points in the cavity using a linear process (e.g. diffraction gratings). In addition the spectral bandwidth has to be filtered by the gain medium to obtain periodic self-consistency $[24,25]$.

For real world applications self-starting and environmentally stable ultrafast allfiber sources are of particular interest. Hence, a linear cavity fiber laser generating self-similar pulses has been developed, which comprises only polarization maintaining (PM) fibers. Two different cavity configurations have been investigated and self-starting self-similar pulses could be generated over a wide range of parameters such as output coupling, pump power and net cavity dispersion.

In Fig. 4.4 the two different cavity configurations can be seen. In both cases the mode-locking mechanism is based on a semiconductor saturable absorber mirror (SAM). Highly efficient transmission gratings are used for intra cavity dispersion compensation. The fiber length inside the cavity was chosen to be the same in the two cases. The only difference is the choice of output coupling. In one case (Fig. 4.4a) the output coupler is a fixed fiber pigtailed coupler allowing for a fiber based output, in the other case (Fig. 4.4b) the output coupler is based on a bulk polarizer and a quarter-wave plate allowing for a tunable coupling ratio. 


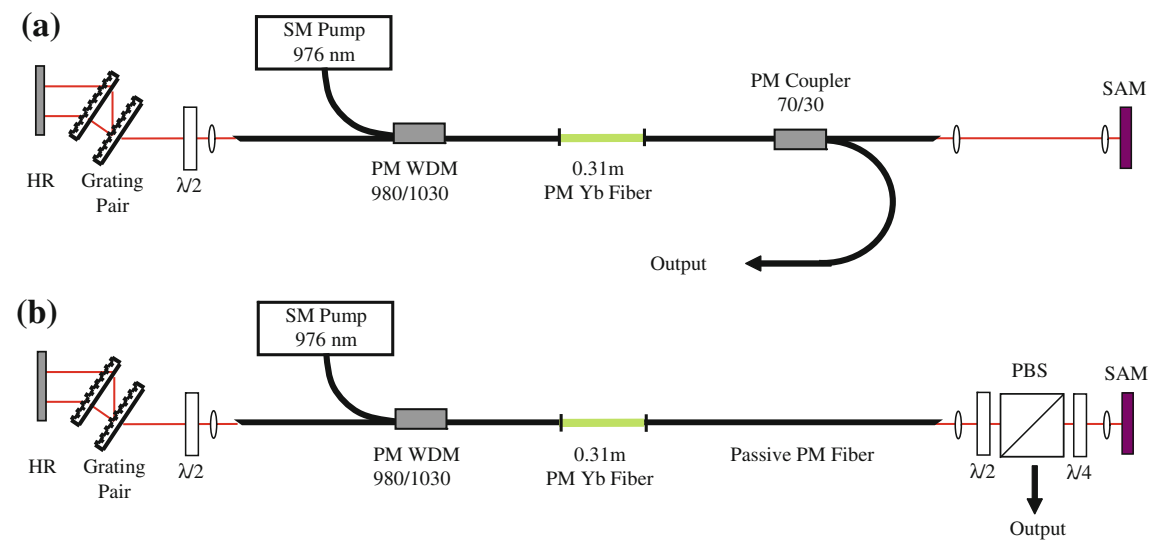

Fig. 4.4 The two different cavity designs: a with fiber output coupler and $\mathbf{b}$ with variable bulk output coupler. $P M$ polarization maintaining, $H R$ high reflection mirror, $S A M$ saturable absorber mirror, $P B S$ polarization beam splitter

The gain medium is a $31 \mathrm{~cm}$ long highly ytterbium doped $(\sim 300 \mathrm{~dB} / \mathrm{m}$ absorption@976 nm) PM fiber with a mode-field diameter of $4.8 \mu \mathrm{m}$. The utilization of the minimal length of gain fiber allows to decouple gain bandwidth filtering from the nonlinear evolution in the undoped fiber because the effect of GVD and nonlinearity can be neglected during the amplification [24]. This fiber is pumped through a thin-film PM wavelength division multiplexer (WDM) by a single mode diode providing a maximum output power of $400 \mathrm{~mW}$ at a wavelength of $976 \mathrm{~nm}$. The passive fibers used in the setup are Panda 980 PM fibers with mode field diameters of $7 \mu \mathrm{m} @ 1035 \mathrm{~nm}$ and a dispersion of $0.024 \mathrm{ps}^{2} / \mathrm{m}$. The total fiber length inside the cavity is $5.6 \mathrm{~m}$ for both cavity designs and in both cases the length of the passive fiber on both sides of the gain medium is equal in order to create symmetric conditions for the pulse evolution after the gain medium in both directions. All the PM fibers were fusion spliced together with an estimated polarization extinction ratio above $37.5 \mathrm{~dB}$ and all fiber ends were either angle polished or angle cleaved. The SAM is commercially available [26] and is based on a non-resonant design, using a GaAs/AlAs Bragg mirror with 27 layer pairs and 26 low temperature molecular beam epitaxy grown InGaAs quantum wells in front of the mirror. The AR coated device has a low-intensity absorption of $45 \%$, a modulation depth of $30 \%$ and a saturation fluence of $\sim 100 \mathrm{~mJ} / \mathrm{cm}^{2}$. In a pumpprobe experiment using 200 fs pulses, the recovery dynamics of the optical excitation has been measured. The SAM shows a bi-temporal impulse response with a short relaxation time of $<200 \mathrm{fs}$ and a slower part of $500 \mathrm{fs}$. The ratio of the fast and slow parts has been determined to 3:2. To achieve the saturation threshold a telescope is used to image the output of the fiber onto the SAM.

The transmission gratings used for intra cavity dispersion compensation are 1250 lines/mm gratings made of fused silica [27] with a high transmission into the first order (>95\%@1035 nm). The gratings are set up in Lithrow angle $\left(40^{\circ}\right)$ with a 
grating separation of $16 \mathrm{~mm}$. A half-wave plate is used between the gratings and the PM fiber to ensure excitation of only the slow axis. In the case of the bulk polarizer and the quarter-wave plate as an output coupler, an additional half-wave plate is also used to ensure excitation of only the slow axis of the fiber after the polarizer. If the axis of the fiber itself is properly aligned to the grating or the polarizer, the halfwave plate can be removed resulting in an even simpler setup. With the fiber pigtailed thin-film 30:70 PM coupler used in the other configuration, a half-wave plate is no longer necessary, as the coupler itself works as a polarizer transmitting only light in the slow axis.

Self-similar spectra, characterized by a parabolic top and with steep edges [24], with a FWHM between 8 and $12 \mathrm{~nm}$ dependent on the pump power, could be obtained with a net cavity dispersion of $0.03 \mathrm{ps}^{2}$. A typical output spectrum is shown in Fig. 4.5, where the FWHM of the self-similar spectrum is $11.3 \mathrm{~nm}$. As the pump power is increased the spectral width increases monotonically, until the threshold for double-pulsing is reached (i.e. two pulses per round trip). The relatively large value of the net cavity dispersion of $\left(\geq 0.03 \mathrm{ps}^{2}\right)$ is chosen to obtain a symmetric spectrum.

In contrast, for lower but still positive values of net cavity dispersion broader, but asymmetric and more structured spectra could be observed, which correspond to the stretched-pulse regime [25]. In the following we will only focus on the selfsimilar regime. The highest output pulse energy is obtained from the setup with the bulk output coupler, where the output coupling could be tuned. For a high output coupling coefficient the highest pulse energies of $1 \mathrm{~nJ}$ are obtained with a repetition rate of $17 \mathrm{MHz}$.

The chirped self-similar output pulse has a duration of $7.2 \mathrm{ps}$ (8.2 ps FWHM on the background free autocorrelator) (see Fig. 4.6), but can be externally compressed to an autocorrelation FWHM of 280 fs. The pulse duration can be calculated from the width of the autocorrelation by assuming a transform limited self-similar

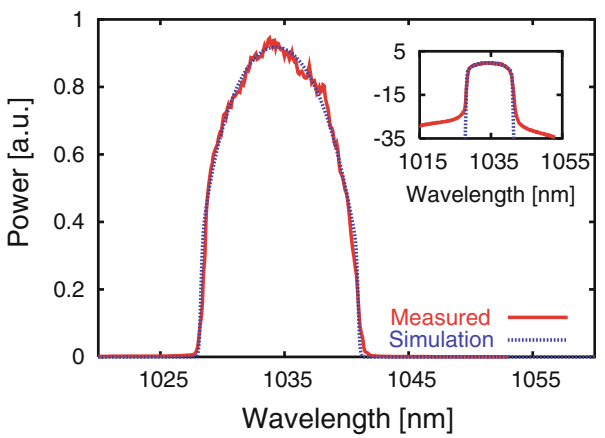

Fig. 4.5 Solid curve Typical output spectrum on a linear scale of the laser operated in the selfsimilar regime (net cavity dispersion $0.03 \mathrm{ps}^{2}$ ). Dotted curve Numerical simulation. Inset Log. scale 


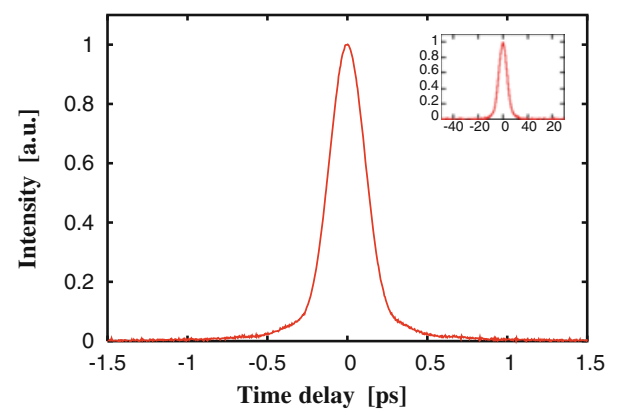

Fig. 4.6 Autocorrelation trace of the externally compressed pulse at a pulse energy of $1 \mathrm{~nJ}$ with an autocorrelation FWHM of 280 fs. Inset Uncompressed output pulse with an autocorrelation FWHM of $8.2 \mathrm{ps}$

spectrum of the compressed pulse (deconvolution factor 1.33) and is evaluated to be 210 fs. From the actual measured spectrum a deconvolution factor of 1.65 is numerically calculated assuming no chirp. It shows that even if the experimental spectrum only slightly deviates from the parabolic shape as in our case, the deconvolution factor changes significantly. Thus, an even more optimistic pulse duration of $170 \mathrm{fs}$ can be evaluated. The scan range of the background free autocorrelator is $150 \mathrm{ps}$, but to check for multiple pulsing and to ensure that there is only a single pulse per round trip, a $25 \mathrm{GHz}$ photo diode in combination with a $50 \mathrm{GHz}$ sampling oscilloscope providing a scan range from 30 to $60 \mathrm{~ns}$ has been used.

Self-similar spectra similar to the spectrum shown in Fig. 4.5 are also obtained from the setup with the fiber pig-tailed coupler. The output pulse energy is $0.12 \mathrm{~nJ}$, the pulse can be externally re-compressed to an autocorrelation FWHM of $350 \mathrm{fs}$. Due to the fixed output coupling of $30 \%$, which is lower than in the other setup, no flexibility is given to find the regime of higher pulse energy and shorter pulses. Additionally, an extra loss of $30 \%$ is introduced inside the cavity due to the fact that the coupler is passed in both directions. Nevertheless, by optimizing the outcoupling ratio it should be possible to obtain better results with the advantage of an alignment-free fiber output.

To make sure the laser operates in the self similar regime some inspections have to be made. Firstly, the spectrum should of course exhibit a self-similar shape, which is fulfilled in our case. Secondly, an important condition for self-similar evolution is that the pulse is always positively chirped inside the cavity, possessing a minimum pulse duration after the intra cavity gratings just before entering the fiber [25]. This is also true since the negative dispersion given by the grating pair used for external compression $\left(-0.36 \mathrm{ps}^{2}\right.$ (double pass)) is higher than of the pair used inside the cavity.

In addition, only half the length of intra cavity fiber is passed before the pulse is coupled out, indicating that the pulse is still highly positively chirped before entering the fiber after the intra cavity grating pair. As the dispersion from this point 
on inside the cavity is positive until the pulse again reaches the intra cavity gratings, this point must be a minimum point. Hence, the pulse is not completely compressed during propagation inside the cavity and, therefore, maintains its shape. As an additional verification, a numerical simulation of the cavity was carried out. Each segment was treated separately by solving the nonlinear Schrödinger equation with the parameters of our experimental setup [24]. In Fig. 4.5 the simulated spectrum can be seen to be in good agreement with the measured spectrum. In the simulation the pulse exhibits self-similar pulse propagation [25] and is always positively chirped.

In both configurations the laser is self-starting and immediately jumps back into the same mode-locked state without any external perturbations if, for instance, switching the laser off and on again. It has been shown, that SAMs exhibiting a bitemporal impulse response can fulfill both requirements of self-starting and symmetric spectra [28]. Furthermore, the use of PM fibers makes the laser stable toward environmentally induced changes to the birefringence of the fiber. The fibers could be twisted and moved around while maintaining a stable modelocked output. This is verified by observing a uniform train of pulses using a fast photo diode and an analogue scope.

The presented approach yields a practical femtosecond laser, which is a perfect seed source for high-power amplifier systems. The parabolic pulse shape is a highly desirable initial condition for further amplification as it helps to avoid excessive accumulation of higher order phase terms along the amplifier chain, which usually degrades the pulse quality and, hence, limits the overall system performance.

The ideal femtosecond laser source for applications outside a laboratory environment would be a completely alignment- and maintenance-free system. Hence, the above laser was developed further to obtain complete fiber integration. The resulting passively mode-locked all-fiber laser is shown schematically in Fig. 4.7. The dispersion compensation is implemented in fiber integrated form as a chirped fiber bragg grating, the saturable absorber mirror is directly glued to the fiber end face. This results in a self-starting, alignment-free turn-key device, which is environmentally stable. Such a compact and robust femtosecond laser is ideally suitable as seed source for compact master oscillator power amplifier systems to be used in rough environments.

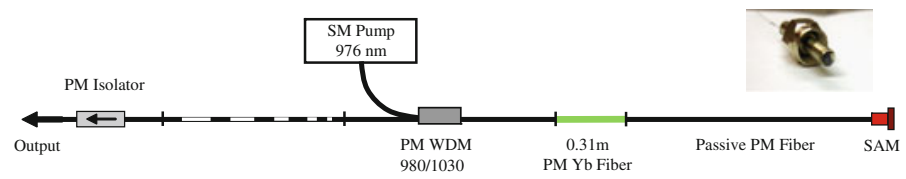

Fig. 4.7 Schematic setup of a passively mode-locker all-fiber oscillator 


\subsection{High Average Power Femtosecond Fiber Amplifier}

In a fiber based CPA system, sufficient pulse stretching and the enlargement of the mode-field diameter of the fiber to reduce the peak power and therefore nonlinear effects such as SRS and SPM are the key points to scale the output parameters. The setup of the state-of-the-art high average power femtosecond fiber laser system is shown in Fig. 4.8 [29]. It consists of a passively mode-locked solid-state laser oscillator, a grating stretcher, a three-stage ytterbium-doped single-mode photonic crystal amplifier and a dielectric reflection-type multi-layer dielectric grating compressor.

The front end oscillator produces $200 \mathrm{fs}$ pulses at $1042 \mathrm{~nm}$ signal wavelength with $78 \mathrm{MHz}$ pulse repetition frequency and $150 \mathrm{~mW}$ average power. The pulses are stretched to $800 \mathrm{ps}$ duration. Afterwards, the remaining signal $(120 \mathrm{~mW})$ is amplified to $50 \mathrm{~W}$ using two amplifier stages that comprise 1.2- and 1.5-m-long double-clad PCFs, both with $40 \mu \mathrm{m}$ core and $170 \mu \mathrm{m}$ pump cladding diameters. All fibers are pumped at $976 \mathrm{~nm}$ wavelength. The main amplifier fiber is a water-cooled 8-m-long double-clad fiber with $26 \mu \mathrm{m}$ mode field diameter and $500 \mu \mathrm{m}$ air clad. This step index fiber has no PCF structure, but the core is nanostructured and consists of $\mathrm{Yb}$ - and F-doped glass rods with sub-wavelength diameters arranged in such a way that they allow tuning the refractive index and, at the same time, reducing the NA. This large mode area fiber was especially designed for high average power operation, i.e. it possesses a large air clad and a signal core that guarantees single mode operation even at this average power levels. The resulting amplifier characteristic is depicted in Fig. 4.9.

At a launched pump power of $1450 \mathrm{~W}$ the signal output power is $950 \mathrm{~W}$ corresponding to $12.2 \mu \mathrm{J}$ pulse energy. The beam quality of the main amplifier was measured to be $\mathrm{M}^{2}=1.3$ at this power level. The resulting maximum compressed signal power is $830 \mathrm{~W}$ with $10.6 \mu \mathrm{J}$ pulse energy. The compression efficiency drops slightly from 95 to $88 \%$ at the maximum signal power because of depolarization taking place inside the fiber. Owing to the acquired nonlinear phase (mainly in the main amplifier) with a calculated B-integral of $11 \mathrm{rad}$, the autocorrelation width

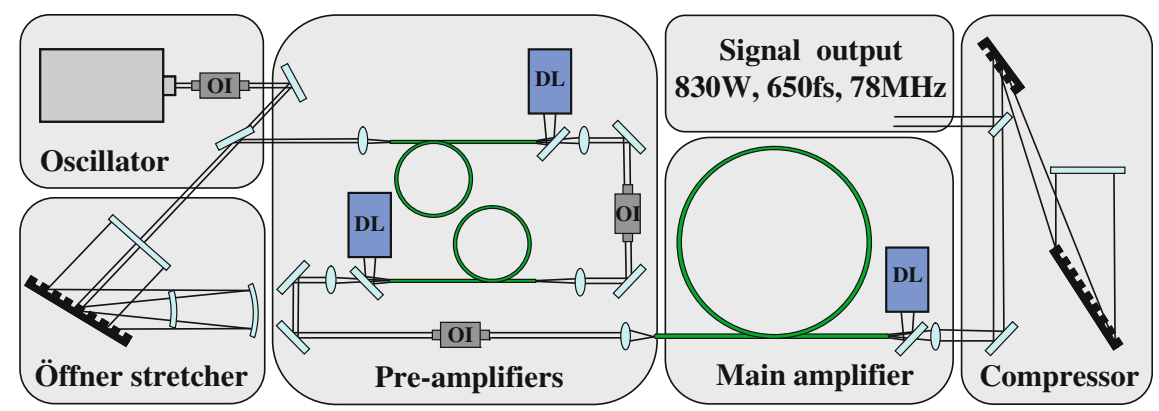

Fig. 4.8 Schematic setup of a high-average power femtosecond CPA system. DL Diode laser, $O I$ optical isolator 
Fig. 4.9 Uncompressed (circles) and compressed (squares) signal average powers depending on the launched pump power

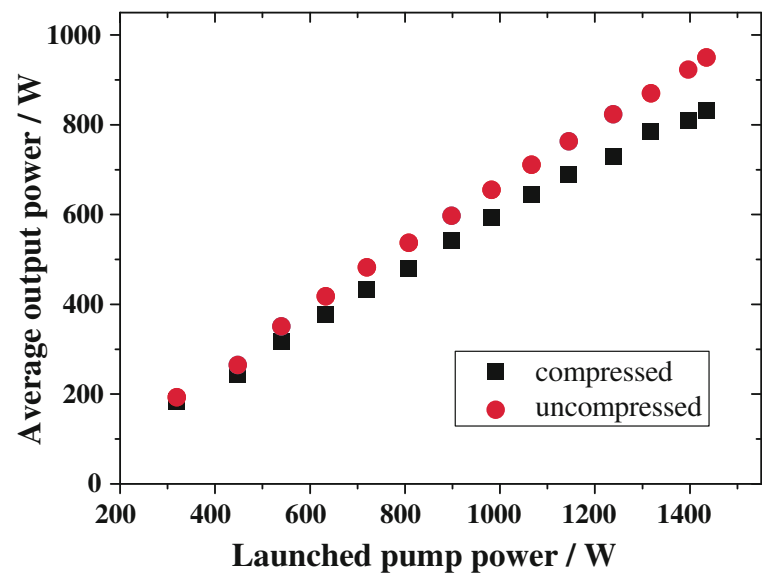

increases from initially 750 to $880 \mathrm{fs}$, the corresponding peak power is approximately $12 \mathrm{MW}$. The setup represents the highest average power among all femtosecond fiber laser systems with close to diffraction-limited beam quality.

\subsection{High Pulse Energy Ultrafast Fiber Amplifier}

Based on the considerations of nonlinearity in fiber chirped pulse amplification systems, an ultrafast high peak power setup has been developed. The schematic setup of the high energy high average power fiber CPA system is shown in Fig. 4.10 [30]. It consists of a passively mode-locked Yb:KGW oscillator, a dielectric grating stretcher-compressor unit, an acousto-optical modulator used as pulse selector and two ytterbium-doped photonic crystal fibers both used in singlepass configuration as amplification stages providing an overall gain factor of approximately 25,000.

The long-cavity $\mathrm{Yb}: \mathrm{KGW}$ oscillator delivers transform-limited $400 \mathrm{fs}$ pulses at a repetition rate of $9.7 \mathrm{MHz}$ with an average power of $1.6 \mathrm{~W}$ at $1030 \mathrm{~nm}$ center wavelength. The stretcher-compressor-unit employs two 1740 lines $/ \mathrm{mm}$ dielectric diffraction gratings, fits on a foot-print of $1.5 \times 0.5 \mathrm{~m}$ and stretches the $3.3 \mathrm{~nm}$ bandwidth pulses to $2 \mathrm{~ns}$. The throughput efficiency of the stretcher is $60 \%$. A quartz based acousto-optical modulator (with a diffraction efficiency as high as $75 \%$ ) is used to reduce the pulse repetition rate.

The pre-amplifier comprises a $1.2 \mathrm{~m}$ long $40 \mu \mathrm{m}$ core single-polarization air-clad photonic crystal fiber having an inner cladding diameter of $170 \mu \mathrm{m}$ pumped by a fiber coupled diode laser emitting at $976 \mathrm{~nm}$. This stage is able of delivering a single-pass gain as high as $35 \mathrm{~dB}$ and average powers up to $6 \mathrm{~W}$, corresponding to a pulse energy of $60 \mu \mathrm{J}$. However, we have operated the preamplifier just up to a few $\mu \mathrm{J}$ of pulse energy to avoid excessive accumulation of nonlinear phase in this stage. The main amplifier is constructed using a $1.2 \mathrm{~m}$ long low-nonlinearity air-cladding 


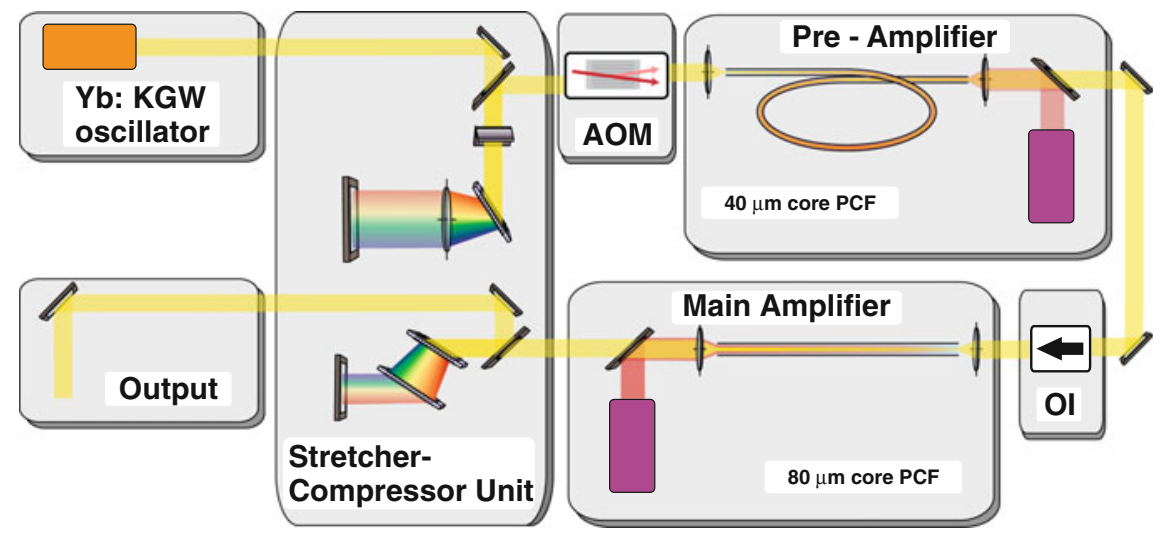

Fig. 4.10 Schematic setup of the $\mathrm{mJ}$ level high repetition rate fiber CPA system; OI optical isolator, $A O M$ acousto-optical modulator

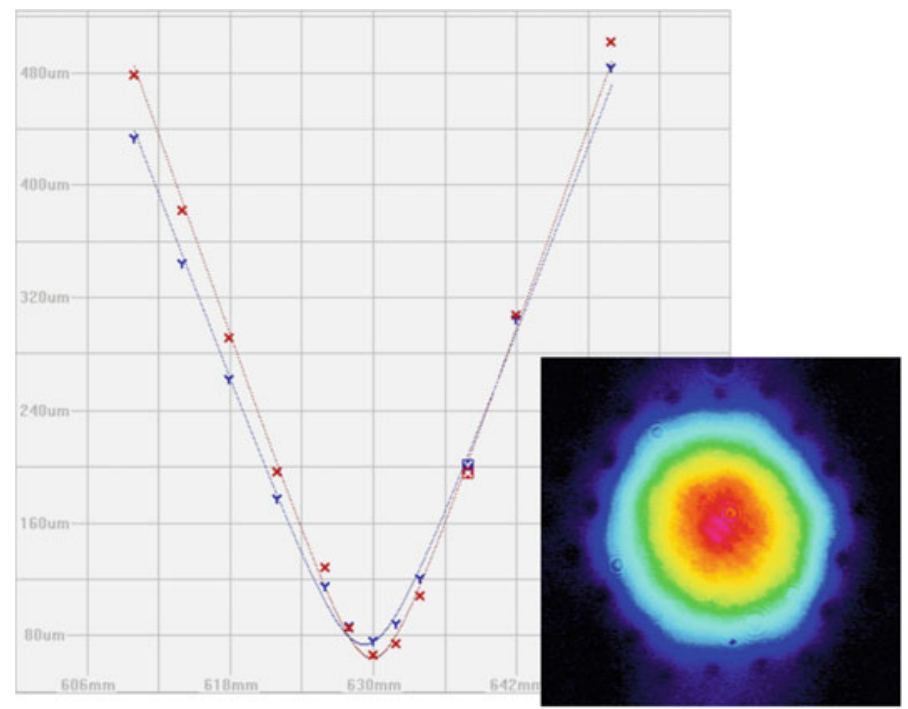

Fig. 4.11 Near-field intensity profile of the $88 \mu \mathrm{m}$ core emission and caustic of the beam quality measurement at $100 \mathrm{~W}$ output power

photonic crystal rod-type fiber as described above. The core supports very few transverse modes; however, stable excitation of the fundamental mode only is achieved by seed mode matching. Figure 4.11 shows a near-field intensity profile of the large-mode area photonic crystal fiber output together with the beam quality characterization measurement. Result is a power independent beam quality characterized by a $\mathrm{M}^{2}$-value of less than 1.2. 
Figure 4.12 shows the output characteristics after compression at $200 \mathrm{kHz}$ repetition rate. The main amplifier was seeded with $0.5 \mathrm{~W}$ of average power, corresponding to $2.5 \mu \mathrm{J}$ pulse energy. At a launched pump power of $230 \mathrm{~W}$ an average output power of $145 \mathrm{~W}$ is obtained with a slope efficiency as high as $66 \%$. Due to the avoidance of any coating material and a stable fiber mounting, no thermo-optical or thermo-mechanical issues are observed up to this average power level. The degree of polarization of the fiber amplifier output is $98 \%$ allowing for an efficient recompression of the pulses. The compressor has a throughput of $70 \%$, leading to a total slope efficiency of $46 \%$ and a compressed average power of $100 \mathrm{~W}$, which implies pulse energies of $500 \mu \mathrm{J}$.

The grating distance in the compressor is always adjusted for minimal autocorrelation width. At $500 \mu \mathrm{J}$ the width is measured to be $1.2 \mathrm{ps}$, as shown in Fig. 4.13 (dashed line), corresponding to a pulse duration of 780 fs assuming a $\operatorname{sech}^{2}$ pulse shape. For comparison the autocorrelation trace at very low pulse energy is also shown (Fig. 4.13, dotted line). The autocorrelation traces reveal a

Fig. 4.12 Compressed average power of the highenergy fiber CPA system at $200 \mathrm{kHz}$ (black squares) and at $50 \mathrm{kHz}$ (red circles)

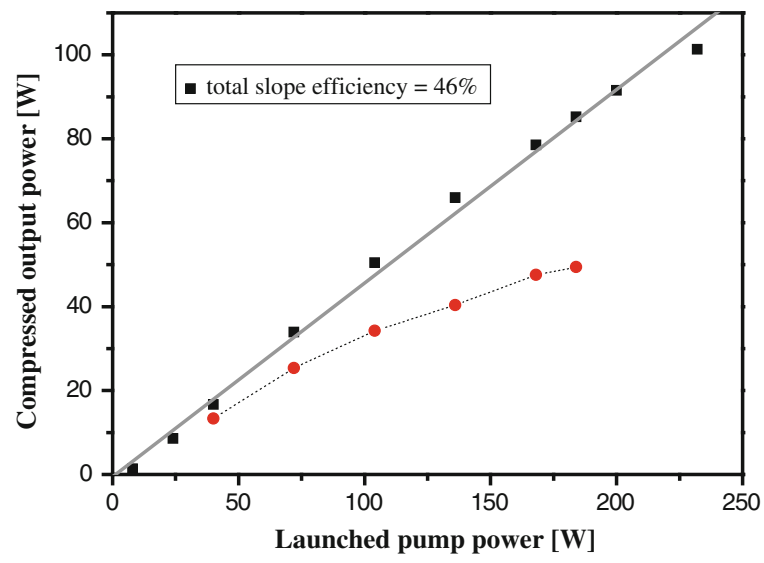

Fig. 4.13 Measured autocorrelation traces of the compressed pulses; dotted at low pulse energy, dashed $200 \mathrm{kHz}$ and $500 \mu \mathrm{J}$, solid $50 \mathrm{kHz}$ and $1 \mathrm{~mJ}$

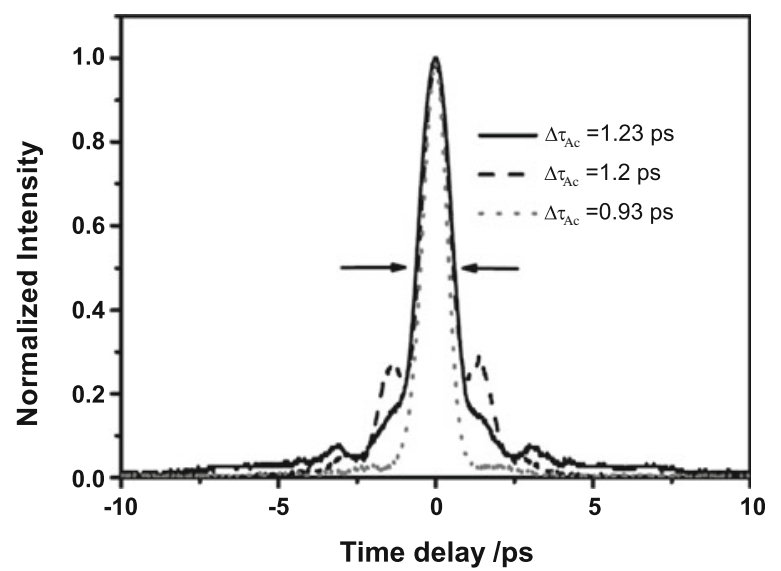


wing structure growing with pulse energy, which can be attributed to the imposed nonlinear phase. The total B-integral is calculated to be $4.7 \mathrm{rad}$ in the case of the $500 \mu \mathrm{J}$ pulses.

Following the discussion above, a further increase of pulse energy without further degradation of pulse quality was possible by decreasing the seed power launched into the main amplifier. On the one hand this reduces the B-integral of the pre-amplifier and, on the other hand the effective nonlinearity of the main amplifier, and therefore the accumulated nonlinear phase per pulse energy, is reduced as well. However, the amplification efficiency is also reduced due to increased pump light absorption saturation, as revealed in Fig. 4.12. At $100 \mathrm{kHz}$ repetition rate, a launched pump power of $220 \mathrm{~W}$ and a seed average power of $100 \mathrm{~mW}$ were necessary to extract $100 \mathrm{~W}$ of average power corresponding to $1 \mathrm{~mJ}$ of pulse energy, leading to $700 \mu \mathrm{J}$ compressed pulse energy. At $50 \mathrm{kHz}$ repetition rate and $70 \mathrm{~mW}$ seed power we achieved $71 \mathrm{~W}$ of average power with a pump power of $180 \mathrm{~W}$, corresponding to $1.45 \mathrm{~mJ}$ energy. In this case the B-integral was $7 \mathrm{rad}$. A further increase in pump power was avoided due to an increased risk of end facet damage and pump light absorption saturation. The compressed pulses exhibited an autocorrelation width of 1.23 ps (equivalent to $800 \mathrm{fs}$ pulse duration), as also shown in Fig. 4.13, and a pulse energy as high as $1 \mathrm{~mJ}$. The stronger nonlinearity is indicated by the wider spread in time of the pedestal. The corresponding pulse peak power is approximately $1 \mathrm{GW}$.

The output spectrum of the high performance fiber CPA system at the highest extracted pulse energy is shown in Fig. 4.14. It is characterized by a $2.9 \mathrm{~nm}$ width (FWHM) and a hard cut of $7.5 \mathrm{~nm}$ bandwidth defined by the stretcher-compressorunit. Only minor gain narrowing is observed for the power levels reported herein. Amplified spontaneous emission and intermediated (non-selected) pulses are suppressed better than $35 \mathrm{~dB}$. The inset of Fig. 4.14 displays a larger wavelength scan of the spectrum showing no evidence of Raman scattering.

Fig. 4.14 Spectral characteristics of the output at $1.45 \mathrm{~mJ}$ pulse energy; inset broader wavelength scan

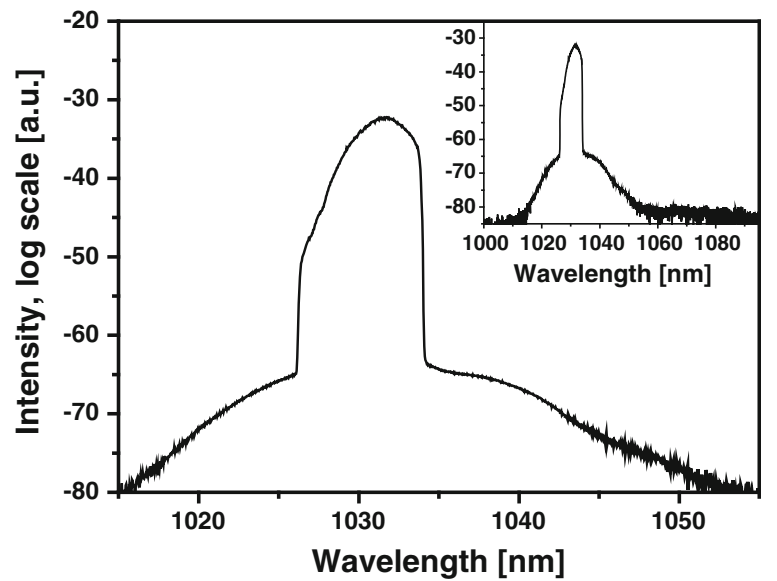




\subsection{Conclusion and Outlook}

Fiber based ultrashort pulse oscillators and amplifiers have reached impressive parameters regarding average power, pulse energy, peak-power and beam quality. Due to this immense progress and their robustness against environmental perturbations they are now ideal sources for fundamental science and industrial production. The further scaling potential is promising and fiber systems delivering femtosecond pulses with average powers beyond $1 \mathrm{~kW}$ and pulse energies of several $\mathrm{mJ}$ will be realized in the near future.

\section{References}

1. A. Giesen, H. Hügel, A. Voss, K. Wittig, U. Brauch, H. Opower, Scalable concept for diodepumped high power solid-state lasers. Appl. Phys. B 58, 365-372 (1994)

2. W.B. Jones, L.M. Goldman, J.P. Chernoch, W.S. Martin, The mini-FPL-a face-pumped laser: concept and implementation. IEEE J. Quantum Electron. 8, 534 (1972)

3. R. Paschotta, J. Nillson, A.C. Tropper, D.C. Hanna, Ytterbium-doped fiber amplifiers. IEEE J. Quantum Electron. 33, 1049 (1997)

4. E. Snitzer, H. Po, F. Hakimi, R. Tumminelli, B.C. McCollum, in Double-clad, offset core Nd fiber laser" in Optical Fiber Sensors, ed. by 1988 OSA Technical Digest Series, vol. 2 (Optical Society of America, Washington, D.C., 1988) postdeadline paper PD5

5. www.ipgphotonics.com

6. G.P. Agrawal, Nonlinear Fiber Optics, 4th edn. (Academic Press, San Diego, 2007)

7. R.R. Alfano, The Supercontinuum Laser Source (Springer-Verlag, New York, 1989)

8. J. Limpert, A. Liem, M. Reich, T. Schreiber, S. Nolte, H. Zellmer, A. Tünnermann, J. Broeng, A. Petersson, C. Jakobsen, Low-nonlinearity single-transverse-mode ytterbium-doped photonic crystal fiber amplifier. Opt. Express 12, 1313-1319 (2004)

9. J. Limpert, O. Schmidt, J. Rothhardt, F. Röser, T. Schreiber, A. Tünnermann, S. Ermeneux, P. Yvernault, F. Salin, Extended single-mode photonic crystal fiber lasers. Opt. Express 14, 2715-2720 (2006)

10. C.D. Brooks, F. Di Teodoro, Multimegawatt peak-power, single-transverse-mode operation of a $100 \mu \mathrm{m}$ core diameter, Yb-doped rodlike photonic crystal fiber amplifier. Appl. Phys. Lett. 89, 111119 (2006)

11. T. Schreiber, F. Röser, O. Schmidt, J. Limpert, R. Iliew, F. Lederer, A. Petersson, C. Jacobsen, K. Hansen, J. Broeng, A. Tünnermann, Stress-induced single-polarization single-transverse mode photonic crystal fiber with low nonlinearity. Opt. Express 13, 7621-7630 (2005)

12. B. Ortaç, O. Schmidt, T. Schreiber, J. Limpert, A. Tünnermann, A. Hideur, High-energy femtosecond Yb-doped dispersion compensation free fiber laser. Opt. Express 15, 1072510732 (2007)

13. J. Limpert, N. Deguil-Robin, I. Manek-Hönninger, F. Salin, T. Schreiber, A. Liem, F. Röser, H. Zellmer, A. Tünnermann, A. Courjaud, C. Hönninger, E. Mottay, High-power picosecond fiber amplifier based on nonlinear spectral compression. Opt. Lett. 30, 714-716 (2005)

14. O. Schmidt, J. Rothhardt, F. Röser, S. Linke, T. Schreiber, K. Rademaker, J. Limpert, S. Ermeneux, P. Yvernault, F. Salin, A. Tünnermann, Millijoule pulse energy Q-switched short-length fiber laser. Opt. Lett. 32, 1551-1553 (2007)

15. H.A. Haus, K. Tamura, L.E. Nelson, E.P. Ippen, Stretched-pulse additive pulse mode-locking in fiber ring lasers: theory and experiment. IEEE J. Quantum Electron. 31, 591-598 (1995) 
16. K. Tamura, L.E. Nelson, H.A. Haus, E.P. Ippen, Soliton versus non-soliton operation of fibre ring lasers. Appl. Phys. Lett. 64, 149-151 (1994)

17. K. Tamura, E. Ippen, H. Haus, L. Nelson, 77-fs pulse generation from a stretched-pulse modelocked all-fiber ring laser. Opt. Lett. 18, 1080-1082 (1993)

18. G. Lenz, K. Tamura, H.A. Haus, E.P. Ippen, All-solid-state femtosecond source at $1.55 \mu \mathrm{m}$. Opt. Lett. 20, 1289-1291 (1995)

19. L. Nelson, S. Fleischer, G. Lenz, E. Ippen, Efficient frequency doubling of a femtosecond fiber laser. Opt. Lett. 21, 1759-1761 (1996)

20. L. Lefort, J. Price, D. Richardson, G. Spühler, R. Paschotta, U. Keller, A. Fry, J. Weston, Practical lownoise stretched-pulse Yb3+-doped fiber laser. Opt. Lett. 27, 291-293 (2002)

21. B. Ortaç, A. Hideur, T. Chartier, M. Brunel, C. Özkul, F. Sanchez, 90 fs generation from a stretched-pulse ytterbium doped fiber laser. Opt. Lett. 28, 1305 (2003)

22. A. Albert, V. Couderc, L. Lefort, A. Barthélémy, High energy femtosecond pulses from an ytterbium doped fiber laser with a new cavity design. IEEE Photon. Technol. Lett. 16, 416418 (2004)

23. F.Ö. Ilday, J. Buckley, H. Lim, F.W. Wise, W. Clark, Generation of 50-fs, 5-nJ pulses at $1.03 \mu \mathrm{m}$ from a wave-breaking-free fiber laser. Opt. Lett. 28, 1365 (2003)

24. F.Ö. Ilday, J.R. Buckley, F.W. Wise, Self-similar evolution of parabolic pulses in a laser. Phys. Rev. Lett. 92, 213902 (2004)

25. J. Buckley, F.Ö. Ilday, F.W. Wise, T. Sosnowski, Femtosecond fiber lasers with pulse energies above 10 nJ. Opt. Lett. 30, 1888 (2005)

26. www.batop.de

27. T. Clausnitzer, J. Limpert, K. Zöllner, H. Zellmer, H.-J. Fuchs, E.-B. Kley, A. Tünnermann, M. Jupé, D. Ristau, Highly efficient transmission gratings in fused silica for chirped-pulse amplification systems. Appl. Opt. 42, 6934-6938 (2003)

28. M. Guina, N. Xiang, A. Vainionpää, O.G. Okhotnikov, T. Sajavaara, J. Keinonen, Selfstarting stretched pulse fiber laser mode locked and stabilized with slow and fast semiconductor saturable absorbers. Opt. Lett. 26, 1809-1811 (2001)

29. T. Eidam, S. Hanf, E. Seise, T.V. Andersen, T. Gabler, C. Wirth, T. Schreiber, J. Limpert, A. Tünnermann, Femtosecond fiber CPA system emitting $830 \mathrm{~W}$ average output power. Opt. Lett. 35, 94-96 (2010)

30. F. Röser, T. Eidam, J. Rothhardt, O. Schmidt, D.N. Schimpf, J. Limpert, A. Tünnermann, Millijoule pulse energy high repetition rate femtosecond fiber chirped-pulse amplification system. Opt. Lett. 32, 3495-3497 (2007) 